blood biomarkers may provide useful information on pulmonary disease burden in both stable and exacerbated states. Serum C reactive protein (CRP) is readily measurable in many clinical settings, including at point-of-care, and procalcitonin (PCT) has a potential role with documented utility in other respiratory diseases. This study explored the relationship between airway and systemic inflammatory biomarkers in adult patients with non-CF bronchiectasis.

Method Serum CRP (for systemic inflammation) and PCT, and sputum interleukin (IL)-6 (for airway inflammation) were measured in 31 well-phenotyped stable patients from The London Bronchiectasis Cohort. Six exacerbation samples were obtained from patients who had been previously sampled at baseline, with the exacerbation sample obtained at exacerbation onset, prior to initiation of additional antibiotic therapy. Sputum IL-6 was measured using ELISA, serum PCT using TRACE, and CRP using immunoturbidimetry techniques. Data were analysed using SPSS V.14.0.

Results Of the 31 patients, 23 (74.2\%) patients were female, with a mean (SD) age of 54.7 (14.9) years, $\mathrm{FEV}_{1} 2.09$ (0.90) 1 $(75.71 \%$ predicted), and FVC 3.12 (1.01) 1. The predominant aetiologies were primary immunodeficiency (mostly CVID, $n=13,42 \%$ ), post-infectious $(10,32 \%)$ and idiopathic (4, 13\%). The median (IOR) baseline sputum IL-6 was 101 (30-701) pg/ml, baseline serum PCT was $0.030(0.021-0.040) \mu \mathrm{g} / \mathrm{ml}$ and CRP $3.0(1.0-6.0) \mathrm{mg} / \mathrm{l}$. The degree of systemic inflammation in stable bronchiectasis reflected that occurring in the airways as evidenced by a significant correlation between sputum IL- 6 and serum CRP in the 31 stable samples $(\mathrm{r}=0.43, \mathrm{p}=0.027)$. There was also a correlation between the two systemic markers (PCT and CRP: $r=0.42, p=0.029$ ), but the relationship between serum PCT and sputum IL- 6 concentration was not statistically significant $(r=0.28, p=0.127)$. In the six patients with paired samples, serum PCT increased significantly from baseline to exacerbation ( 0.031 vs $0.147 \mu \mathrm{g} / \mathrm{ml} \mathrm{p}=0.043$ ). Changes in sputum IL-6 and blood serum CRP did not reach statistical significance.

Conclusion We report a direct relationship between airway and systemic inflammation in stable patients with non-CF bronchiectasis. This suggests that systemic biomarkers may be useful for monitoring local disease activity in this neglected condition.

\section{P247 THE RESPIRATORY PHENOTYPE OF THE BBS4 NULL MOUSE LUNG}

doi:10.1136/thoraxjnl-2011-201054c.247

${ }^{1} \mathrm{M}$ Cho, ${ }^{2} \mathrm{P}$ Beales, ${ }^{1} \mathrm{M}$ Hind, ${ }^{2} \mathrm{R}$ Quinlan. ${ }^{1}$ Imperial College, London, UK; ${ }^{2}$ University College London, London, UK

Introduction Bardet-Biedl Syndrome (BBS) is an autosomal recessive disorder due to BBS gene mutations that result in primary ciliary dysfunction (Hildebrandt et al, N Engl J Med 2011). Primary cilia are found on most eukaryotic cells and have been reported in various human organs including kidneys, brain, and lung airways. BBS patients present with a number of features including polydactyly, obesity, visual impairment and renal cysts and these are also seen in other examples of ciliopathies. Many BBS patients report frequent respiratory infections and are empirically diagnosed with asthma; yet, little is known about the pulmonary effects of BBS and explanations for the respiratory presentations remain unclear. We have recently described the respiratory phenotype of a cohort of 28 consecutive BBS patients (Hind: unpublished data). This study investigates whether there are structural and functional defects in the BBS lung that may explain the frequent respiratory symptoms described by BBS patients.
Methods Lungs from Bbs4 null mice (Bbs4-/-) were examined and compared to unaffected control littermates. Lung volumes were measured by volume displacement and alveolar tissue assessed through morphometry according to ATS/ERS standards (Hsia, AJRCCM, 2010). These data were used to derive the alveolar surface area (Sa) for each animal. Functional studies of motile cilia using ciliary beat frequency $(\mathrm{CBF})$ and inflammatory cell types in the distal lungs were analysed both from bronchoalveolar lavage fluid (BALF) and using immunohistochemistry on lung sections

Results We show that, as seen in the heterogeneous patient group, the Bbs4-/- mice are significantly heavier than control littermates. Lung volume and Sa of all mice increase with age; however, Bbs4-/ - mice have smaller lung volumes and a reduced Sa. Furthermore, lung histology demonstrates that Bbs4-/- mice show evidence of chronic infection. Although we have not found any evidence for ultrastructural defects in airway motile cilia, Bbs4-/- null mice have a significantly slower CBF.

Conclusion These data suggest that a loss of function in BBS4 results in a greater body mass, a functional motile ciliary defect, frequent inflammation, and reduced gas-exchanging surface area of the lung.

\section{P248 ASSOCIATIONS OF DEPRESSION, FATIGUE AND QUALITY OF LIFE IN ADULT NON-CF BRONCHIECTASIS}

doi:10.1136/thoraxjnl-2011-201054c.248

H Batchelor, S Brill, J S Brown, J R Hurst. UCL Medical School, London, UK

Introduction Non-cystic fibrosis bronchiectasis is a chronic lung condition characterised by persistent cough, excessive sputum and recurrent chest infections. Symptoms also include dyspnoea, haemoptysis, depression and fatigue. The latter can have a major impact on patients' physical and psychosocial well-being. We investigated disease- and patient-related factors associated with quality of life, fatigue and depression in bronchiectasis.

Method Patients were recruited from the London Bronchiectasis Cohort. A clinical diagnosis of bronchiectasis was confirmed by review of previous CT imaging. A full medical history including exacerbations and aetiology, spirometry, and the SGRQ (for quality of life), CESD (depression), and FACIT (fatigue) questionnaires were completed at a baseline appointment. Statistics were analysed using SPSS.

Results 31 patients (25 female) were studied with a mean (SD) age of 58.8 (SD 12.0) years. The commonest aetiologies were postinfectious (15/31) and idiopathic (11/31). The median (IOR) selfreported exacerbation frequency was 3.0 (1.9-5.6)/year.

Frequent exacerbations were associated with poorer quality of life: there was a significant association between exacerbation frequency and the SGRQ score $(\mathrm{r}=0.46, \mathrm{p}=0.013)$. Frequent exacerbations were also associated with greater baseline breathlessness $(\mathrm{r}=0.40, \mathrm{p}=0.038)$, but not with lung function, depression or fatigue. Patients with greater disease severity (lower $\mathrm{FEV}_{1}$ ) also had more breathlessness (MRC: $\mathrm{r}=-0.38, \mathrm{p}=0.046$ ), and poorer quality of life (SGRQ: $r=-0.50, p=0.005$ ) but not greater depression or fatigue. The depression scale was not related to any of the disease or patient-related factors. The only patient or disease-related variable associated with fatigue was breathlessness (MRC: $r=-0.57$, $\mathrm{p}=0.002$ ).

Conclusion The determinants of quality of life, depression and fatigue in non-CF bronchiectasis are different. Depression in this population appears to be independent of markers of disease severity such as $\mathrm{FEV}_{1}$, exacerbation frequency and MRC dyspnoea score. 